ESAIM: PROCEEDINGS, October 2011, Vol. 32, p. 231-241

E. Cancès, N. Crouseilles, H. Guillard, B. Nkonga, and E. Sonnendrücker, Editors

\title{
INFLUENCE OF WAVES ON LAGRANGIAN ACCELERATION IN TWO-DIMENSIONAL TURBULENT FLOWS ${ }^{*, * *, * * *}$
}

\author{
Romain Nguyen van yen ${ }^{1}$, Benjamin Kadoch ${ }^{2}$, Vivek KumaR ${ }^{1}$, Benjamin \\ Ménétrier $^{1}$, Marie Farge ${ }^{1}$, Kai Schneider ${ }^{2}$, Diane Douady ${ }^{3}$ and Lionel Guez ${ }^{1}$
}

\begin{abstract}
The Lagrangian statistics in rotating Saint-Venant turbulence are studied by means of direct numerical simulation using a pseudo-spectral discretization fully resolving, both in time and space, all the inertio-gravity waves present in the system. To understand the influence of waves, three initial conditions are considered, one which is dominated by waves, one which is dominated by vortices, and one which is intermediate between these two extreme cases. It is found that Lagrangian acceleration is strongly influenced by the presence of waves, as are the Lagrangian velocity increments, for incremements that are sufficiently short, and that vortex motion influences mostly the component of Lagrangian acceleration which is perpendicular to the local velocity.
\end{abstract}

\section{INTRODUCTION}

Among the many kinds of motion occuring in multiscale flows, the case of waves that are both fast enough and small enough in amplitude is perhaps the best understood, since these waves can be described by expanding the equations in terms of different time scales, a technique known as fast-slow splitting in singular perturbation theory [2]. The rotating Saint-Venant equations (also called shallow water equations) in the limit of small Rossby number, i.e., for a referential rotating at a frequency larger than the flow vorticity, have been a toy model for developing such approaches in weakly nonlinear regimes and understanding their properties. We refer the reader to the textbook [16] for a self-contained tutorial on the subject. In the context of rotating Saint-Venant, the splitting allows to average out fast inertio-gravity waves. As first realized by Rossby [12], in an appropriate parameter regime the slow component is described to first order in Rossby number by the quasigeostrophic equations, which are completely independent on the behavior of the fast component. In particular,

\footnotetext{
* The authors thank the CIRM Marseille for its hospitality and for financial support during the CEMRACS 2010 Summer Program where part of the work was carried out, and IDRIS-CNRS for providing computing facilities.

** VK would like to thank the French embassy in India for financial support of his visit in ENS Paris, and EGIDE for all kind of help.

*** RNVY, MF and KS are thankful to the Association CEA-EURATOM and to the French Research Federation for Fusion Studies for supporting their work within the framework of the European Fusion Development Agreement and the French Research Federation for Fusion Studies under the contract V.3258.001. This work is supported by the European Communities under the contract of Association between Euratom and CEA. The views and opinions expressed herein do not necessarily reflect those of the European Commission.

1 LMD-CNRS, ENS, 24 rue Lhomond, 75231 Paris Cedex

2 M2P2-CNRS \& CMI Universités d'Aix-Marseille

3 Département de Mathématiques, Université de Cergy
} 
this usually requires that the wave amplitude parameter, denoted $\lambda$ from now on, is of the same order as the Rossby number.

The aim of the work presented in this paper is to understand the effect of such waves on passively advected pointwise massless particles, in the case where the vortical flow is in the fully developed turbulence regime, which is the most difficult to address analytically, since the separation of time scales between the waves and the vortical flow becomes only marginally valid. As a first approach to this problem we choose the rotating Saint-Venant equations, which are among the simplest models giving rise to both waves and turbulent motion. They are classically used to describe geophysical flows at the synoptic scale, since the Earth's curvature and the non-hydrostatic vertical motions can then be neglected. In atmospheric models, which aim to predict large scale flows, it is essential to take into account gravity waves [9]. It has been shown [6] that both rotation and inertiogravity waves reduce the nonlinear interactions between geostrophic vortices and thus inhibit the turbulent cascade for scales larger than the Rossby deformation radius. New ad-hoc models are still being actively developed to improve over existing approximations [7], and it is thus important to improve our understanding of the effect of waves in this context. Other long term motivations for this work are magnetic confinement fusion which involves fast drift waves [5], and solar physics where fast magneto-gravity waves enter into play [13].

A famous example of impact of the waves on particles is the Stokes drift [14], which is formally of second order in terms of the wave amplitude. More comprehensive studies of the retroaction of the waves on mean Lagrangian quantities have been performed since then (see e.g. [3]). On the other hand, the behavior of the fast field may also be interesting for its own sake, in particular when considering quantities that are sensitive to high frequency flow variations. The Lagrangian acceleration, on which we focus in this work, is one of them. We closely follow an approach which was previously applied to 2D incompressible turbulence [10] and 2D drift-wave turbulence [11]. As can be seen from a naive order of magnitude estimate, although the particle displacement due to fast waves may be negligible because it is proportional to $\lambda$, the acceleration experienced by a particle during the passage of a wave is proportional to $\omega^{2} \lambda$ (where $\omega$ is a characteristic wave frequency) which is sure to become non-negligible for fast enough waves. However, as we shall see below, statistics previously observed in incompressible turbulence can be recovered in rotating Saint-Venant turbulence at the price of some time integration, or by direct observation of the Lagrangian velocity.

In the following, the rotating Saint-Venant equations are solved by direct numerical simulation, and to maintain a high-level of turbulence the flow is initialized with random fields excited on a wide range of scales, and hyperdissipation is used to widen the inertial range. No external forcing term is applied to the flow. Several numerical simulations are performed with different levels of inertio-gravity waves and two rotation rates. To advect the particles, Eulerian velocity is computed at each time step, and bicubic interpolation is used. We first report on the properties of the flows in the Eulerian reference frame as has been classically considered, and then we proceed with the results concerning the Lagrangian trajectories, which are the original part of our work. Finally we draw some overall conclusions.

\section{Method}

\subsection{Dynamical model}

To tackle this type of problems, we adopt an ab initio approach, which consists in solving numerically the full rotating Saint-Venant system with spatial and temporal resolutions that are sufficiently fine to resolve all the waves. The rotating Saint-Venant equations describe the flow of a thin incompressible fluid layer which rotates at a constant rate and satisfies the following additional hypotheses:

- the fluid is in hydrostatic equilibrium with gravity,

- all vertical gradients are negligible compared to horizontal gradients,

- the thickness of the water layer is negligible compared with its characteristic horizontal size. 
Under these hypotheses, conservation of mass and momentum imply the following system:

$$
\left\{\begin{aligned}
\lambda \partial_{t} h+\nabla \cdot(\mathbf{u}(1+\lambda h)) & =0 \\
\partial_{t} \mathbf{u}+(\mathbf{u} \cdot \boldsymbol{\nabla}) \mathbf{u}+\frac{1}{\mathrm{Ro}} \widehat{\mathbf{z}} \times \mathbf{u}-\frac{\lambda}{k_{d}^{2} \mathrm{Ro}^{2}} \nabla h & =0
\end{aligned}\right.
$$

where $\mathbf{u}(\mathbf{x}, t)$ is the velocity field, the space variable $\mathbf{x}$ varies in a horizontal domain, $\widehat{\mathbf{z}}$ is the upward unit vector normal to this domain, $h$ is the surface height perturbation, Ro is the Rossby number, $k_{d}$ is the Rossby deformation wavenumber and $\lambda$ is the nonlinearity parameter. Since the nonlinearities are bound to excite ever smaller spatial scales, we have to include some kind of dissipation mechanism to compensate. For this we employ the technique of hyperdissipation, both for the velocity equation and for the height perturbation equation, which consists in adding a term $c \Delta^{8} \mathbf{u}$ (respectively $c \Delta^{8} h$ ) on the right hand side of the respective equations for $\mathbf{u}$ and $h$. Compared to a standard dissipative term, this classical procedure [1] allows for a wider inertial range by concentrating dissipation at higher wavenumbers. To approximate homogeneous isotropic turbulence, the equations are solved in the periodic domain $\mathbb{T}^{2}=(\mathbb{R} / \mathbb{Z})^{2}$. The choice of units that we have made leads to the following link between the dimensionless numbers appearing in (1) and physical quantities: Ro $=U L^{-1} f^{-1}$, and $\lambda=\Delta H H_{0}^{-1}$, where $U$ is a characteristic velocity constructed from the total initial energy, $k_{d}=L f\left(g H_{0}\right)^{-1 / 2}, f / 2$ is the rotation frequency, $g$ is the modulus of gravity, $H_{0}$ is the height of the fluid at rest, $L$ is the size of the domain, and $\Delta H$ is the initial maximum height perturbation. Time is normalized by the eddy turnover time $L U^{-1}$.

In the absence of dissipation $(c=0)$, a remarkable property of solutions to the Saint-Venant equations is that the potential vorticity

$$
q=\frac{\mathrm{Ro}^{-1}+\zeta}{1+\lambda h}
$$

is conserved along Lagrangian trajectories, where $\zeta=\boldsymbol{\nabla} \times \mathbf{u}$ is the vorticity of the flow in the rotating frame of reference (sometimes called relative vorticity). Moreover, these solutions have constant energy

$$
E=\frac{1}{2} \int_{\mathbb{T}^{2}}\left((1+\lambda h) \mathbf{u}^{2}+\frac{\lambda k_{d}^{-2}}{\mathrm{Ro}^{2}} h^{2}\right),
$$

and constant potential enstrophy

$$
Z_{p}=\frac{1}{2} \int_{\mathbb{T}^{2}} \frac{\left(\mathrm{Ro}^{-1}+\zeta\right)^{2}}{1+\lambda h}
$$

Since the energy is not a quadratic form in terms of the flow variables, the classical techniques of spectral analysis used in turbulence cannot be directly applied. A quadratic invariant is recovered only in the limit where the Saint-Venant equations can be linearized around a rest state where velocity $\mathbf{u}=0$, and the height perturbation vanishes. In this case, a convenient way of analyzing the flow consists in expanding it over the eigenmodes of this linearized system, which are given in Fourier space by:

$$
\widehat{\mathbf{u}}_{0}(\mathbf{k})=\frac{i \mathbf{k}_{\perp}}{k_{d}^{2}+\mathbf{k}^{2}}, \quad \widehat{h}_{0}(\mathbf{k})=\frac{k_{d}^{2} \operatorname{Ro} \lambda^{-1}}{k_{d}^{2}+\mathbf{k}^{2}}, \quad \widehat{\mathbf{u}}_{ \pm}(\mathbf{k})=k_{d} \frac{ \pm \mathbf{k} \sqrt{k_{d}^{2}+\mathbf{k}^{2}}-i \mathbf{k}_{\perp}}{2 \mathbf{k}^{2}\left(k_{d}^{2}+\mathbf{k}^{2}\right)} \text { and } \widehat{h}_{ \pm}(\mathbf{k})=\frac{\operatorname{Ro} \lambda^{-1} k_{d}^{2} \mathbf{k}^{2}}{2 \mathbf{k}^{2}\left(k_{d}^{2}+\mathbf{k}^{2}\right)}
$$

where $\mathbf{k}$ is the wavenumber, and $\mathbf{k}_{\perp}$ is obtained by rotating $\mathbf{k}$ by $\frac{\pi}{2}$. In the following, the projecton of the flow over the eigenmodes indexed by 0 will be called "potentio-vortical flow", while the residual part of the flow, corresponding to the projection over the eigenmodes indexed by \pm , will be called "inertio-gravitational", Since the eigenmodes are orthogonal, the total energy can be split in the same way into potentio-vortical and inertio-gravitational energies. In the nonlinear regime, the potentio-vortical and inertio-gravitational energies are no longer constant, but the separation can still be carried out and yields information about the properties of the flow [6], which is meaningful as long as $\lambda$ remains small. 
A remarkable property of the rotating Saint-Venant equations is the tendency of their solutions, under some conditions, to approximately satisfy the geostrophic balance relation:

$$
\mathbf{u} \approx\left(\begin{array}{c}
-\partial_{y} \\
\partial_{x}
\end{array}\right) \frac{\lambda h}{k_{d}^{2} \mathrm{Ro}} .
$$

This relation can also be visualized, as we shall do below, using the streamfunction $\psi$. The streamfunction $\psi$ and the velocity potential $\chi$ are both uniquely defined up to a constant by the Helmholtz decomposition of $\mathbf{u}$ :

$$
\mathbf{u}=\widehat{\mathbf{z}} \times \nabla \psi+\nabla \chi
$$

and geostrophic balance then corresponds to

$$
\psi \approx \frac{\lambda h}{k_{d}^{2} \mathrm{Ro}} \text { and } \quad \chi \approx 0 .
$$

The rotating Saint-Venant equations are discretized spatially using a pseudo-spectral method [4], and temporally using the leap-frog scheme, in order to preserve the dispersive properties of the inertio-gravity waves. All the results presented below were obtained in double precision and with a cut-off wavenumber of spatial discretization equal to 128 , which amounts to using a collocation grid having $N=256$ in each direction, or equivalently a mesh size $\frac{\pi}{128}$. The duration of the timestep is fixed throughout the computation to a value which ensures numerical stability according to a CFL-type criterion based on the phase velocity of the fastest resolved waves. Note that the numerical difficulty of this problem comes mostly from the shortness of the timesteps that are required to accurately resolve all the inertio-gravity waves.

\subsection{Particle advection}

In the following we consider $N_{p}=1024$ pointwise massless particles, also called Lagrangian tracers, that are being advected by the flow without inertia effects. The trajectory $\mathbf{X}_{i}(t)$ of each of these particles is governed by the following equation:

$$
\frac{d \mathbf{X}_{\mathbf{i}}}{d t}:=\mathbf{v}_{i}(t)=\mathbf{u}\left(\mathbf{X}_{i}(t), t\right)
$$

where $\mathbf{v}_{i}(t)$ is, by definition, the Lagrangian velocity of the particle, and $\mathbf{u}\left(\mathbf{X}_{i}(t), t\right)$ is the Eulerian velocity at the particle position, which needs to be interpolated using the values given by the Eulerian solver at each timestep on the colocation grid. For this we choose to use bicubic interpolation, and (6) is then advanced in time using a second-order Runge-Kutta scheme.

The main goal of this work is the study of the Lagrangian acceleration $\mathbf{a}_{i}=\frac{d^{2} \mathbf{x}}{d t^{2}}$, which can also be obtained by interpolation from the Eulerian quantities thanks to the formula:

$$
\frac{d^{2} \mathbf{X}_{i}}{d t^{2}}=\frac{D \mathbf{u}}{D t}=-\frac{1}{\mathrm{Ro}} \widehat{\mathbf{z}} \times \mathbf{u}+\frac{\lambda k_{d}^{-2}}{\mathrm{Ro}^{2}} \nabla h
$$

where $\frac{D}{D t}=\frac{\partial}{\partial t}+u_{1} \frac{\partial}{\partial x_{1}}+u_{2} \frac{\partial}{\partial x_{2}}$ is the material derivative, and the dissipative term has been neglected. In the following we shall also consider the Lagrangian velocity increments

$$
\Delta \mathbf{v}_{i}(t, \tau)=\mathbf{v}_{i}(t+\tau)-\mathbf{v}_{i}(t)
$$

which are related to $\mathbf{a}_{i}$ by

$$
\Delta \mathbf{v}_{i}(t, \tau)=\int_{t}^{t+\tau} \mathbf{a}_{i}\left(t^{\prime}\right) \mathrm{d} t^{\prime}
$$


and in particular, assuming sufficient regularity of $\mathbf{v}_{i}(t)$ :

$$
\mathbf{a}_{i}(t)=\lim _{\tau \rightarrow 0} \Delta \mathbf{v}_{i}(t, \tau) .
$$

We now describe the Lagrangian statistical quantities that will be presented below. The main idea is to accumulate statistics over the full ensemble of particles and as much time as possible, so that convergence can be ensured at a reasonnable computational cost. However, since the flow decays in time, the statistics of the Lagrangian velocity and acceleration are not stationary. First, the initial period of time where there is a rapid relaxation of the enstrophy (see Fig. 1) is eliminated by considering the statistics only for $t>t_{C}$, where the values of $t_{C}$ will be specified later. Then, to obtain data with constant standard deviation for $t>t_{C}$, we normalize at each time $\mathbf{v}_{i}(t)$ by $\sigma_{v}(t)=\left(\sum_{i=1}^{N_{p}} \mathbf{v}_{i}(t)^{2}\right)^{1 / 2}$, and $\mathbf{a}_{i}(t)$ by $\sigma_{a}(t)=\left(\sum_{i=1}^{N_{p}} \mathbf{a}_{i}(t)^{2}\right)^{1 / 2}$. This procedure was suggested by [15], and has been applied in previous work, for example in [10]. We denote by $\tilde{\mathbf{v}}_{i}$ and $\tilde{\mathbf{a}}_{i}$ the normalized quantities. Note that (9) still holds for the normalized quantities.

From $\tilde{\mathbf{v}}_{i}, \Delta \tilde{\mathbf{v}}_{i}$ and $\tilde{\mathbf{a}}_{i}$ we shall construct probability density functions (PDFs), approximated by agregating histograms in time with 100 fixed bins. Since the problem is isotropic, we limit ourselves, without loss of generality, to the first component of each quantity. We shall also consider the velocity increments flatness:

$$
F(\tau)=\frac{\int \mathrm{d} t \sum_{i=1}^{N_{p}}\left|\Delta \tilde{\mathbf{v}}_{i}(t, \tau)\right|^{4}}{\left(\int \mathrm{d} t \sum_{i=1}^{N_{p}}\left|\Delta \tilde{\mathbf{v}}_{i}(t, \tau)\right|^{2}\right)^{2}} .
$$

\subsection{Parameters}

Three cases are considered to obtain the results presented below.

First, a wave-dominated flow, for which the initial velocity field is zero, and the initial height perturbation is randomly distributed (with a relative maximum amplitude of 1 , as required by our choice of units), and we choose $\lambda=0.01$ as nonlinarity parameter. The Fourier coefficients of the initial height perturbation field are of the form $k^{\alpha} e^{i \theta}$, where $\theta$ is a pseudo-random number uniformly distributed in the interval $[0,2 \pi], k$ is the modulus of wavenumber, $\alpha=3$ for $k \leq k_{e}$ and $\alpha=-3$ for $k>k_{e}$. Here, $k_{e}$ is the excitation wavenumber, which we shall take equal to 8 . These Fourier coefficients correspond to an initial potential energy spectrum which is proportional to $k^{7}$ for $k \leq k_{e}$ and to $k^{-5}$ for $k>k_{e}$. Since potential vorticity (2) is preserved along the flow trajectories, and initially has the form

$$
q=\frac{f}{1+\lambda h}
$$

this initial condition will lead to the propagation of inertio-gravity waves, but will also generate a small amount of vorticity. For this case the Rossby number is taken equal to $1.26 \cdot 10^{-3}$, and the deformation wavenumber to $k_{d}=2$.

Next, a vortex-dominated flow is considered. A random height perturbation is first generated using the above method. The Rossby number and deformation wavenumber are fixed respectively to Ro $=4.11 \cdot 10^{-2}$ and $k_{d}=2$, and the flow that would be in geostrophic equilibrium with this perturbation at $\lambda=0.01$ is then computed and chosen as initial condition for $\mathbf{u}$. However, the value of $\lambda$ which is actually used when solving the equation is taken as $\lambda=0.05$, which is an ad hoc way of pushing the flow out of geostrophic equilibrium and thus generate inertio-grativy waves. As a result of this procedure, the initial inertio-gravitational energy equals $2 \%$ of the total initial energy.

For the third case, the same procedure is applied except that the Rossby number is reduced by a factor 6 . As a consequence, the amount of waves in the initial condition is increased, which is why we call this case "mixed flow". In this case, the initial inertio-gravitational energy thus equals about $28 \%$ of the total initial energy.

The parameters corresponding to the three types of flows just described are recalled in Table 1 . The flows were integrated for as much time as could computationally be afforded, given the smallness of the timestep 


\begin{tabular}{|c|c|c|c|c|c|}
\hline & Ro & $k_{d}$ & $k_{e}$ & $\lambda$ & $c$ \\
\hline Wave-dominated (a) & $1.26 \cdot 10^{-3}$ & 2 & 8 & 0.01 & $10^{-37}$ \\
Vortex-dominated (b) & $4.11 \cdot 10^{-2}$ & 2 & 8 & 0.05 & $1.3 \cdot 10^{-39}$ \\
Mixed (c) & $1.55 \cdot 10^{-3}$ & 12 & 8 & 0.05 & $10^{-38}$ \\
\hline
\end{tabular}

TABLE 1. Parameters of numerical experiments.

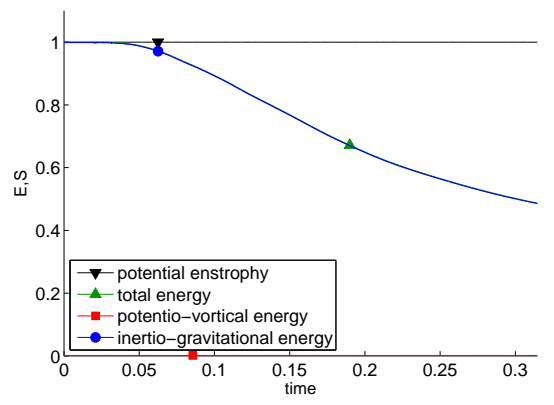

(a) Wave-dominated

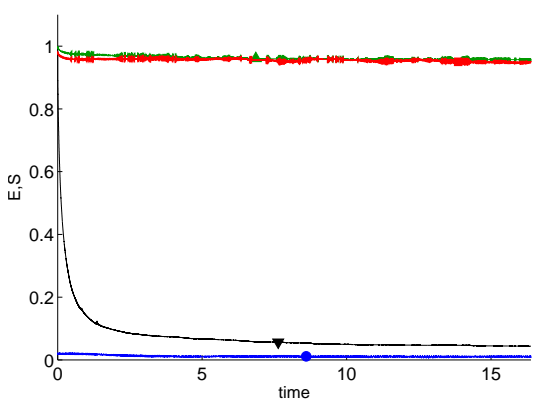

(b) Vortex-dominated

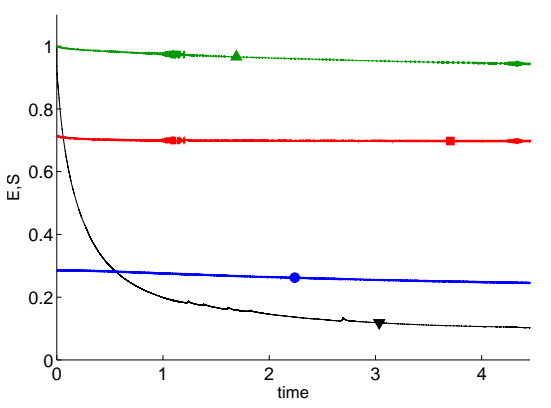

(c) Mixed

FiguRE 1. Time evolution of potential enstrophy, and of the various components of energy as defined by the splitting defined in Sec. 2.1. Energy components are normalized by the total initial energy, and potential enstrophy by its initial value.

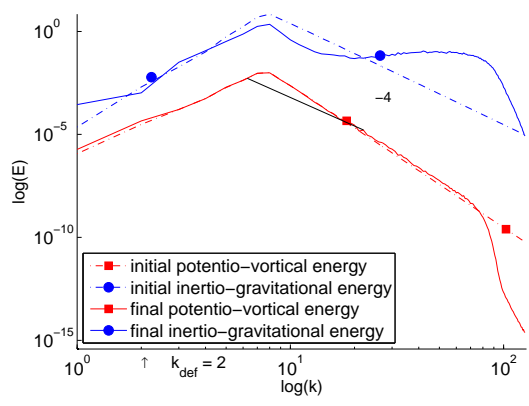

(a) Wave-dominated

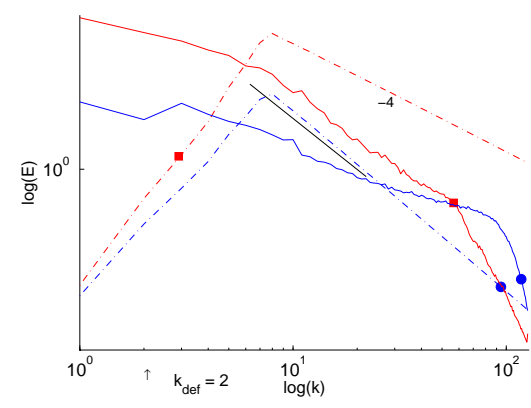

(b) Vortex-dominated

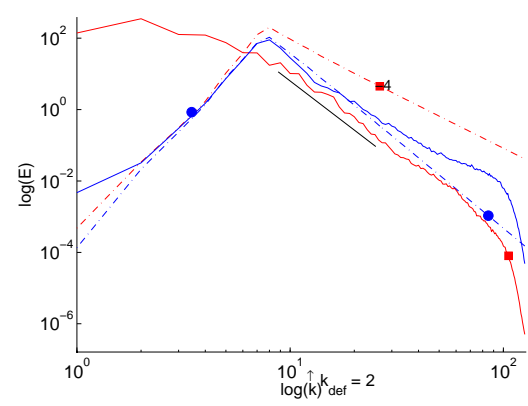

(c) Mixed

Figure 2. Initial and final potentio-vortical and inertio-gravitational energy spectra.

required to temporally resolve all the waves. Thus, the final times that we reached were $t=0.315, t=16.4$ and $t=4.45$, for the wave-dominated, vortex-dominated and mixed cases, respectively.

\section{Results on Eulerian QuANTIties}

\subsection{Wave dominated flow}

The time evolution of the various energy components is plotted in Fig. 1 (left). The energy consists mostly of inertio-gravitational energy, which slowly decays during the flow evolution. The corresponding initial and final energy spectra are shown in Fig. 2. It is seen that the inertio-gravitational energy spreads towards high frequency, in a range of wavenumbers comprised roughly between 10 and 100 . This can be mostly attributed to the nonlinear three-wave interactions, since the vortical part remains negligible and doesn't spectrally evolve. 


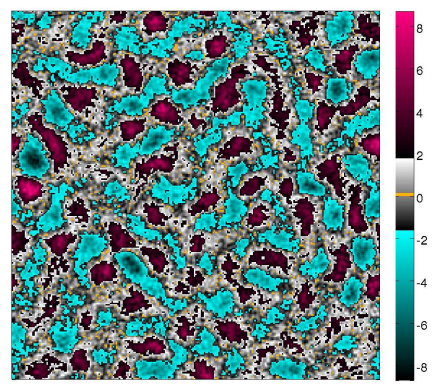

(a) Wave-dominated

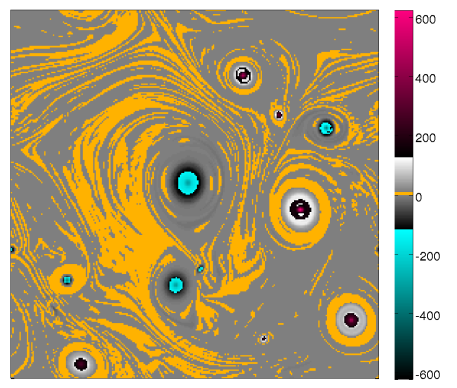

(b) Vortex-dominated

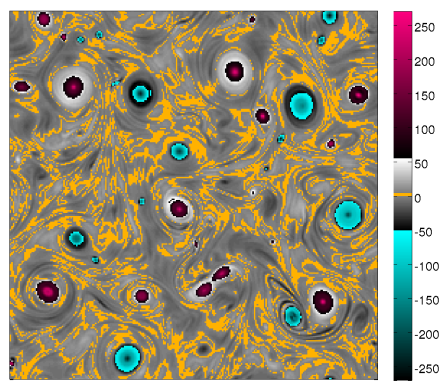

(c) Mixed

Figure 3. Potential vorticity snapshots at final time (Left to right: $t=0.315, t=16.4, t=4.45$ ).

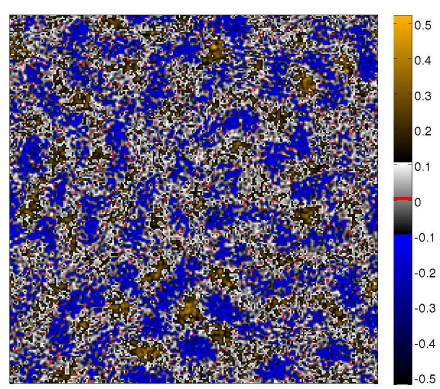

(a) Wave-dominated

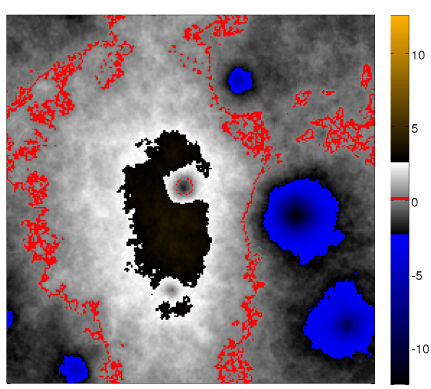

(b) Vortex-dominated

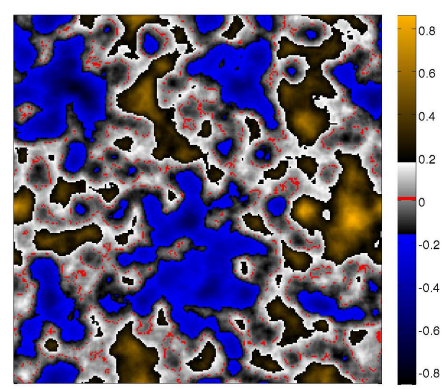

(c) Mixed

Figure 4. Height perturbation snapshots at final time (Left to right: $t=0.315, t=16.4, t=4.45$ ).

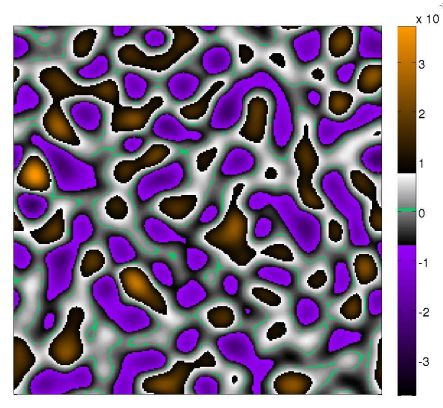

(a) Wave-dominated

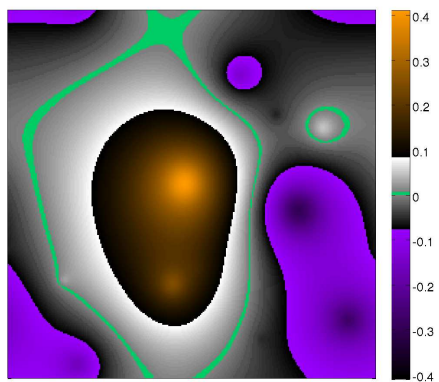

(b) Vortex-dominated

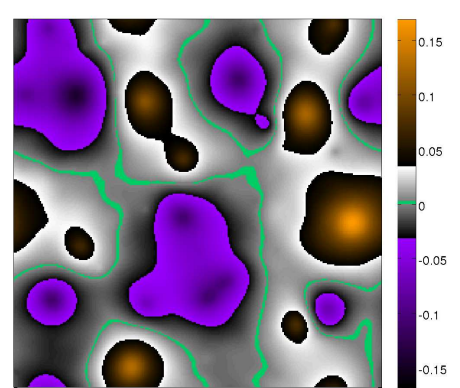

(c) Mixed

Figure 5. Stream function snapshots at final time (Left to right: $t=0.315, t=16.4, t=4.45$ ). 


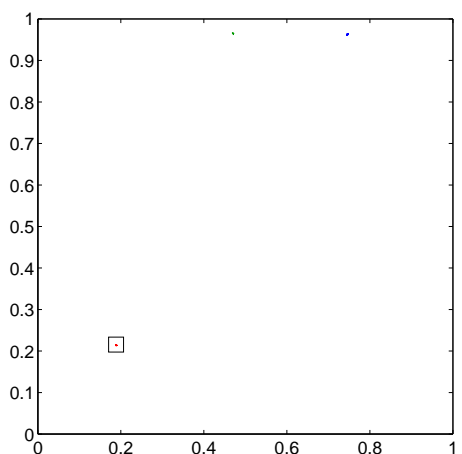

(a) Wave-dominated

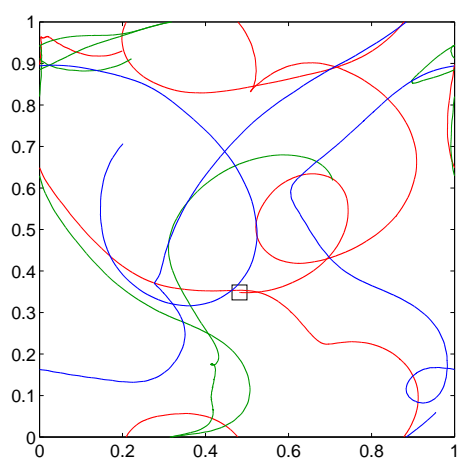

(b) Vortex-dominated

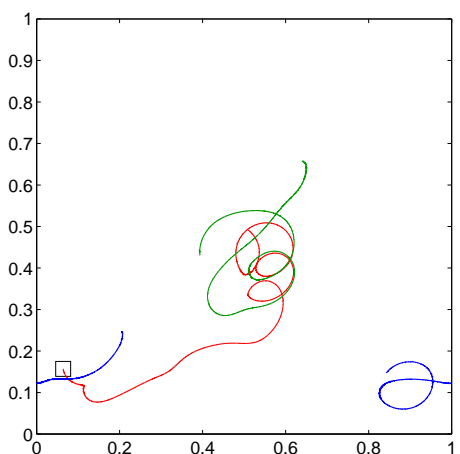

(c) Mixed

FigurE 6. Trajectories of three particles chosen randomly of the 1024 available in total.

The potential vorticity, height perturbation, and stream function fields are represented respectively in Figs. 3 and 4, 5 (left). No coherent structures are visible in the potential vorticity field, and the height perturbation and stream function do not show any sign of geostrophic equilibrium, which would correspond to the relationship (5) between $\psi$ and $h$. The motion remains ageostrophic in this case.

\subsection{Vortex dominated flow}

Energy (Fig. 1, middle) is almost conserved, while potential enstrophy is rapidly dissipated at the beginning of the considered time interval. The final potentio-vortical energy spectrum (Fig. 2, middle) has its maximum at $k=1$, indicating that a notable amount of energy has been transfered to lower wavenumbers by an inverse cascade process. In the range $8 \leq k \leq 80$, a $k^{-4}$ spectrum has established itself as a consequence of the nonlinear flow evolution. The inertio-gravitational energy spectrum has become noticeably flatter than in the initial condition, and seems to have a slope lying between -1 and -2 . No spurious accumulation of energy at high wavenumber is visible on these spectra, which indicates that the computations are well resolved.

Distinct coherent vortices are visible in the final potential vorticity field (Fig. 3, middle). By comparing the final height perturbation and stream function (Figs. 4 and 5, middle), it can be seen that geostrophic equilibrium is satisfied at large scales (according to (5)). In addition to its large scale geostrophic features, the height perturbation field contains fine scale oscillations which can be attributed to the presence of gravity waves.

\subsection{Mixed flow}

The energy (Fig. 1, right) decays more than in the previous case, and this decay is mostly due to the dissipation of inertio-gravitational energy. The inertio-gravitationnal energy spectrum (Fig. 2, right) has not evolved much between the initial and final times. In contrast, the potentio-vortical energy spectrum shows that an inverse energy cascade has occured for the vortices.

\section{Results on Lagrangian Statistics}

To reduce the impact of the initial condition on the Lagrangian statistics, they are analyzed only for $t>t_{C}$, where $t_{C}=0.192$ for the wave-dominated case, $t_{C}=8$ for the vortex-dominated case, and $t_{C}=2.25$ for the mixed case. The trajectories of three particles are shown in Fig. 6. In the left panel, corresponding to the 


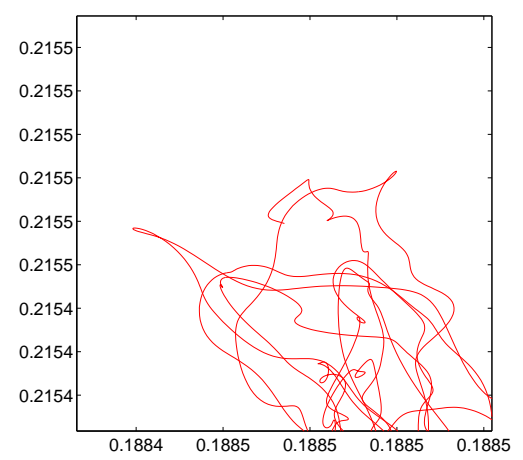

(a) Wave-dominated

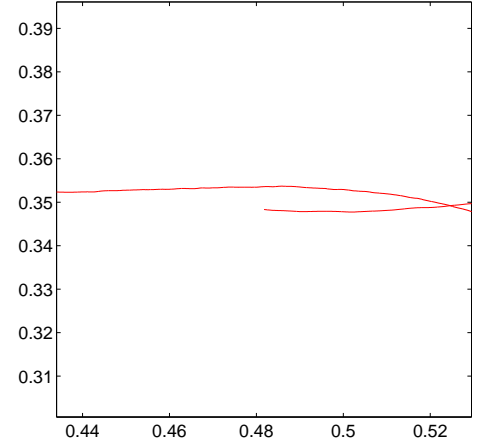

(b) Vortex-dominated

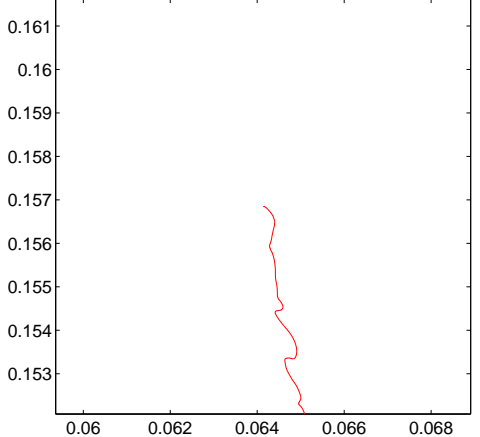

(c) Mixed

Figure 7. Zoom on the beginning of the trajectory of a particle, in the regions indicated by the black square in Fig. 6. The enlargement factors are respectively $2 \cdot 10^{4}, 20$ and 200 .

wave-dominated flow, the trajectories are barely visible. To enhance the tiny motion of the particle, we show a zoom in Fig. 7.

The main result of this work consists in the PDFs shown in Fig. 8. In each graph, the PDFs of the first component of Lagrangian velocity and of Lagrangian acceleration are plotted along with the PDFs of the first component of Lagrangian velocity increments for varying increment size. The various PDFs have been shifted vertically in order to better distinguish between them, with the acceleration always located at the top, the velocity at the bottom, and the increments in the middle. The PDFs are also rescaled to have their standard deviation equal to 1 . The first thing we notice is that the PDFs of velocity increments converge to the PDFs of Lagrangian acceleration when the increments goes to zero, in agreement with (9).

For the wave-dominated flow (Fig. 8, a), all the PDFs are very nearly similar to each other, suggesting that the trajectories are self-similar. The PDFs are also close to being Gaussian, as indicated by the fact that their flatness (Fig. 8, d) is nearly equal to 3. For the vortex-dominated flow (Fig. 8, b), the PDF of the Lagrangian acceleration is non Gaussian and has an inflection point around 3 standard deviations. The core of the PDF, in the interval $[-3,3]$, is reminiscent ot the wave-dominated case, but the wings extend further away from zero, up to 7 standard deviations. This effect is reflected on the PDFs of Lagrangian velocity increments for $\Delta t \leq 0.01$. In constrast, from $\Delta t=0.05$ upwards, the wings take more and more importance, and the PDFs resemble those that were observed in incompressible $2 \mathrm{D}$ turbulence in [10], with values reaching 10 standard deviations. For even larger $\Delta t$, as in [10], the wings lose weight again and the Lagrangian velocity PDF is closer to a Gaussian. These transitions are reflected by the behavior of the flatness (Fig. 8, d), which first raises from 7 to 30 and then goes back down to around 7 at large increments. In the mixed case (Fig. 8, c), which contains more inertio-gravitational energy than the wave-dominated case, the PDF of Lagrangian acceleration is very close to the one which was observed in the wave-dominated case (Fig. 8, a). Only for time increments larger than 3.95 can the effect of the vortices be detected on the PDF of velocity increments and on its flatness.

Another way of outlining the effect of the waves is to decompose the Lagrangian acceleration over the local Frenet frame attached to the particle, or in other words to project it along the directions that are parallel and orthogonal to the local Lagrangian velocity. The resulting PDFs, shown in Fig. 9, are almost overlapping in the wave-dominated and in the mixed-case, but they are markedly different in the vortex-dominated case. More precisely, in the vortex-dominated case, the tails of the perpendicular acceleration PDF extend further away from the origin, while the PDF of parallel acceleration remains Gaussian. This interesting effect is in fact not very surprising, since it means that the effect of the vortices is mostly to bend the trajectories of the particles. 


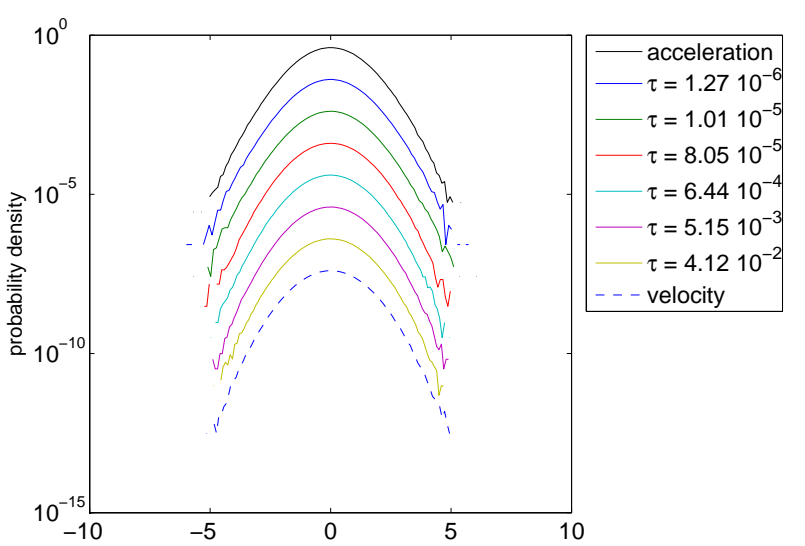

(a) Wave-dominated

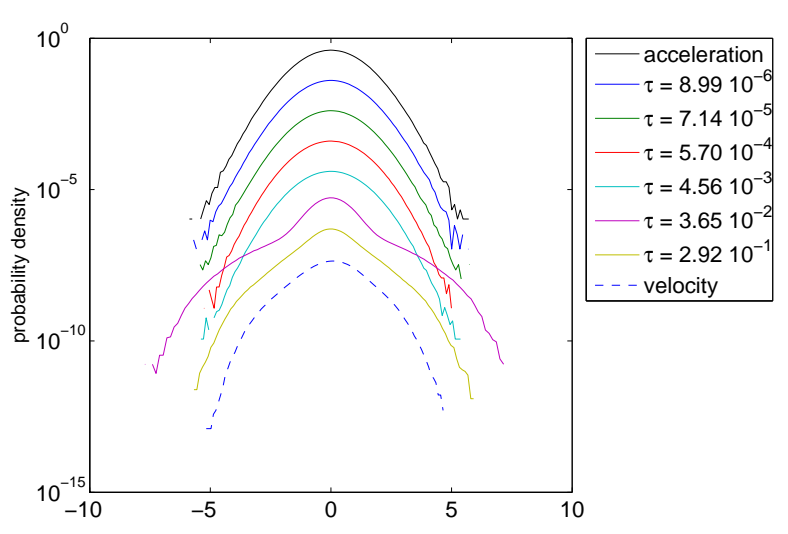

(c) Mixed

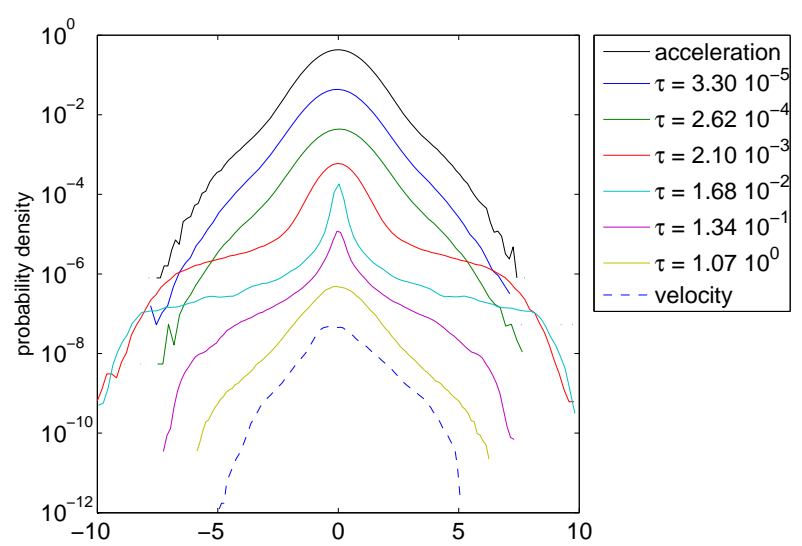

(b) Vortex-dominated

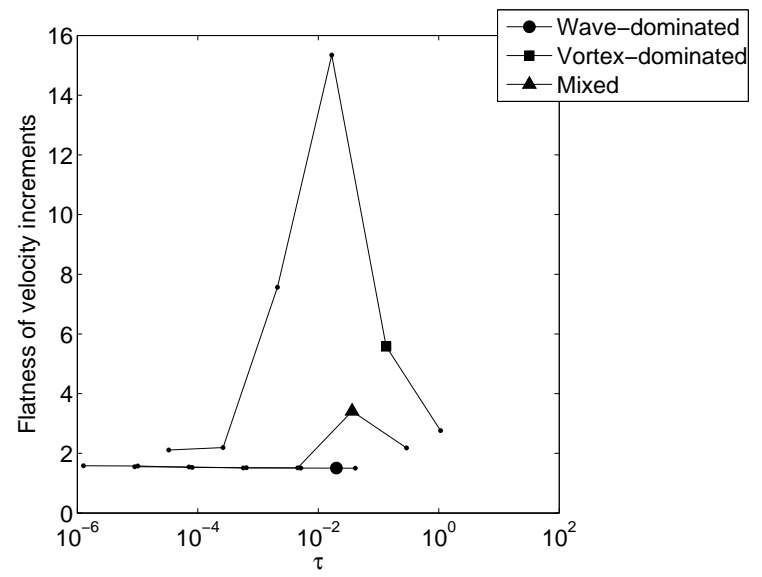

(d)

FIGURE 8. (a-c) PDFs of the first component of Lagrangian velocity, Lagrangian velocity increments, and Lagrangian acceleration (see Sec. 2.2). The standard deviation is normalized to one, and the curves have been shifted vertically for better visualization. (d) Flatness of Lagrangian velocity increments $\mathbf{v}(t+\tau)-\mathbf{v}(t)$ as a function of $\tau$ for the three types of flows.

\section{Conclusion}

In this work, we have studied the Lagrangian statistics of rotating Saint-Venant turbulence by direct numerical simulation, in order to shed some light on the effect fast waves can have on these quantities. We have shown that the PDFs of Lagrangian quantities, such as velocity increments and acceleration, were strongly influenced. The extent of this influence is determined both by the amplitude of the waves and by their frequency. At least for short enough increments, the core of the velocity increments PDFs seems to be mostly determined by the waves. This result could have implications on the interpretation of experimental results concerning particles advected by geophysical flows in the atmosphere or ocean. More generally, the Lagrangian point of view we have proposed may help characterize the wave content of experimental or numerical flows using probes such as drifting buoys or balloons [8]. This work is a first step towards a more comprehensive study of turbulent 


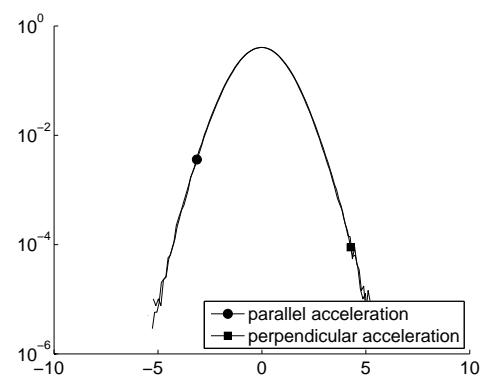

(a) Wave-dominated

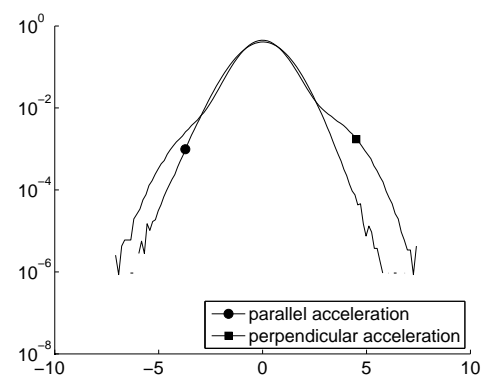

(b) Vortex-dominated

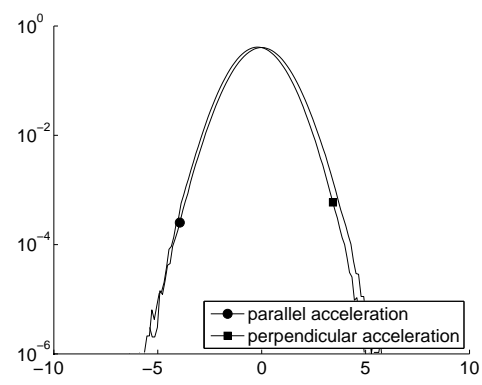

(c) Mixed

FiguRE 9. PDFs of the parallel and perpendicular components of the Lagrangian acceleration relative to the local particle velocity.

dissipation in many types of turbulent flows affected by fast waves, as encountered for example in geophysics or plasma physics.

\section{REFERENCES}

[1] C. Basdevant, B. Legras, R. Sadourny and M. Béland. A study of barotropic model flows: intermittency, waves and predictability. J. Atmos. Sci., 38, (1981), 2305-2326.

[2] C. M. Bender and S. A. Orszag. Advanced mathematical methods for scientists and engineers I: asymptotic methods and perturbation theory. Springer (1999).

[3] O. Bühler and M. E. McIntyre. On non-dissipative wave-mean interactions in the atmosphere or oceans. J. Fluid Mech., 354, (1998), 301-343.

[4] C. Canuto, A. Quarteroni, M. Y. Hussaini and T. A. Zang. Spectral methods in fluid dynamics. Springer-Verlag (1988).

[5] P. H. Diamond, C. J. McDevitt, Ö. D. Gürcan, T. S. Hahm and V. Naulin. Transport of parallel momentum by collisionless drift wave turbulence. Physics of Plasmas, 15, no. 1, (2008), 012303-+.

[6] M. Farge and R. Sadourny. Wave-vortex dynamics in rotating shallow water. J. Fluid Mech., 206, (1989), 433-462.

[7] D. C. Fritts and M. J. Alexander. Gravity wave dynamics and effects in the middle atmosphere. Rev. Geophys., 41, (2003), $1003-+$.

[8] R. R. Harcourt, E. L. Steffen, R. W. Garwood and E. A. D'Asaro. Fully Lagrangian Floats in Labrador Sea Deep Convection: Comparison of Numerical and Experimental Results. J. Phys. Oceanogr., 32, (2002), 493-510.

[9] C. O. Hines. Internal atmospheric gravity waves at ionospheric heights. Can. J. Phys., 38, (1960), 1441-+.

[10] B. Kadoch, W. J. T. Bos and K. Schneider. Extreme lagrangian acceleration in confined turbulent flow. Phys. Rev. Lett., 100, (2008), 184503.

[11] B. Kadoch, W. J. T. Bos and K. Schneider. Origin of Lagrangian intermittency in drift-wave turbulence. Phys. Rev. Lett., 105, (2010), 145001.

[12] C. G. Rossby. On the mutual adjustment of pressure and velocity distributions in certain simple current systems, II. J. Mar. Res., 2, (1938), 239-263.

[13] D. A. Schecter, J. F. Boyd and P. A. Gilman. "Shallow-Water" Magnetohydrodynamic Waves in the Solar Tachocline. Astrophysical J., 551, (2001), L185-L188.

[14] G. Stokes. On the theory of oscillatory waves. Trans. Cambridge Phil. Soc., 8, (1847), 441.

[15] P. K. Yeung. Lagrangian investigations of turbulence. Annu. Rev. Fluid Mech., 34, (2002), 115-142.

[16] V. Zeituin, ed. Nonlinear dynamics of rotating shallow water. Elsevier (2007). 